\title{
Magnetic Resonance and CT Imaging Biomarkers for Prediction of Acute and Chronic Radiation-Induced Xerostomia
}

\author{
Anastasia Katsavochristou ${ }^{1, *(\mathbb{D})}$ and Dimitrios Koumoulis ${ }^{2, *}$ \\ 1 Division of Prosthodontics, University of Kentucky College of Dentistry, Lexington, KY 40536, USA \\ 2 Center for Applied Energy Research, University of Kentucky, Lexington, KY 40511, USA \\ * Correspondence: anastasia.katsavochristou@uky.edu (A.K.); dimitrios.koumoulis@uky.edu (D.K.)
}

Citation: Katsavochristou, A.;

Koumoulis, D. Magnetic Resonance and CT Imaging Biomarkers for Prediction of Acute and Chronic Radiation-Induced Xerostomia. Magnetochemistry 2021, 7, 5. https://dx.doi.org/10.3390/ magnetochemistry7010005

Received: 1 November 2020 Accepted: 21 December 2020 Published: 24 December 2020

Publisher's Note: MDPI stays neutral with regard to jurisdictional claims in published maps and institutional affiliations.

Copyright: () 2020 by the authors. Licensee MDPI, Basel, Switzerland. This article is an open access article distributed under the terms and conditions of the Creative Commons Attribution (CC BY) license (https: / / creativecommons.org/ licenses/by/4.0/).

\begin{abstract}
Xerostomia is a common adverse effect of radiation therapy at the head and neck area. Radiation-induced xerostomia can be severe and detrimental for the quality of life. Clinicians and radiologists have focused on the prevention of xerostomia as feasible, which has been significantly improved in the recent decades with the use of the contemporary radiation technology. However, radiation-induced xerostomia still remains one of the most devastating side effects of radiation therapy. Clinical risk factors have been identified, but the variation of its incidence and presentation has turned the focus on the investigation of parameters that would be able to predict the onset of acute or chronic xerostomia for each individual patient. Recently, potential imaging parameters and biomarkers are investigated in order for early prediction of the incidence and severity of xerostomia. Here, we compile the resulting imaging biomarkers as have been identified in the recent literature based on MRI and CT performed in correlation with radiation therapy. The identification of such biomarkers is very promising for the prevention and control of xerostomia in the head and neck radiation setting.
\end{abstract}

Keywords: xerostomia; radiation therapy; magnetic resonance; MRI; CT

\section{Introduction}

Oncologic pathology can be often a greatly devastating finding with consequences in multiple aspects of the individual such as physical, psychological, social, and financial. The consequences can be long term, and, thus, affecting the quality of life of the individual $[1,2]$. The latter is the principal consideration for the multidisciplinary care team, along with the survival of the patient. This becomes very important as, with even the most successful treatment of cancer in regards to survival, the quality of life can often remain significantly compromised not only due to the destruction that the oncologic pathology caused but also due to the necessary for survival treatment(s) itself.

A common detrimental short- and long-term effect of a cancer treatment is the postradiation therapy-induced xerostomia ("dry mouth"), strongly associated with radiation therapy of the head and neck area and, in particular, of the oropharyngeal and nasopharyngeal areas [3-5]. Xerostomia is a very common side effect due to the destructive effect of radiation on the highly radiosensitive salivary glands. It is radiation field and dose dependent, and it can start to be noticed not only during the radiation therapy but also long term in the everyday life of the patient. The reduction in the saliva directly affects a multitude of oral and physical functions (e.g., mastication, deglutition, and speech), and it can consequently affect nutrition, psychology, and social interactions of the individual. The severity increases when the major salivary gland, the parotid gland, is in the field of radiation.

Salivary glands undergo physiologic changes due to radiation. A recent study found that the preradiation imaging of the parotid and submandibular glands has the potential to predict postradiation acute xerostomia. In specific, the glands were depicted as being more hypodense, hypointense, and heterogeneous in the baseline CT and MRI imaging 
of the patients that developed moderate or severe xerostomia 3 months after completion of the radiation therapy [6]. The incidence of chronic (1 year after radiation therapy) radiation-induced xerostomia in head and neck cancer patients and various primary tumor sites was found to be $49 \%$ for xerostomia grade $1,14 \%$ for grade 2 , and $2 \%$ for grade 3 . Notably, $83.3 \%$ of this patient population had received concurrent chemotherapy [7].

Treatment of established radiation-induced xerostomia is challenging in order to reestablish normal function. Hence, of paramount importance for the effective management of xerostomia is prevention, as feasible, and early diagnosis in order to promote and improve intervention and management. Prevention can be feasible through the radiotherapy planning phase of the radiation target areas, but also through a readjustment of the individualized planning during the radiation treatment.

Biomarkers and imaging analysis can provide scientific data that, when characterized by increased sensitivity and specificity, have a great potential for pretreatment risk analysis, early diagnosis, staging, monitoring, and evaluation of the effectiveness of an intervention. Otherwise, diagnosis and monitoring could be only reached by clinical assessment of established signs and symptoms of the patient that can be expressed with a different rate for every patient. Delaying a diagnosis correlates with a more established biologic and physiologic underlying entity, which usually correlates, in turn, with a less efficient management.

Here, we review the current status of the role of magnetic resonance imaging and computed tomography for the assessment of radiation-induced xerostomia based on specific imaging biomarkers and parameters, as reflected in the literature for the years 2010-2020.

\section{Computed Tomography (CT) and Radiation-Induced Xerostomia}

\subsection{Acute Xerostomia}

Information deriving from CT imaging of the parotid glad throughout radiotherapy has been analyzed in order to evaluate the use of specific CT data for early prediction of radiation-induced xerostomia. In particular, the combination of volume changes and imaging texture analysis of the parotid, as reflected by the fractal dimension data, was found to provide the highest predictability of $71.4 \%$ for the parotid gland changes between the first and the last week of radiation therapy (Table 1) [8]. These results were based on a 6-8 weeks IMRT radiotherapy of the nasopharynx, where the parotid glands were not tumor involved areas.

A more recent study attempted to correlate specific CT parameters with the observed xerostomia, as evaluated at each week of the radiation therapy. A correlation could not be concluded between dose-volume parameters and xerostomia grade. However, a predictability of xerostomia grade in the range of 79-98\% was found when a model was used that was based on both the volume change and changes of the mean CT number (MCTN), as reported once a week throughout the treatment (Table 1). Additionally, for a subset of patients, when the difference of the values of the aforementioned two parameters between the first fraction and fifth week were applied in the model, the resulting xerostomia grade at the fifth week was predicted $100 \%$ [9]. These results are very promising, as the combination of the two imaging derived parameters, volume and MCTN, are showing quantitative correlation with the xerostomia grade during each week of the radiation treatment. 
Table 1. Imaging biomarkers for acute and chronic radiation-induced xerostomia.

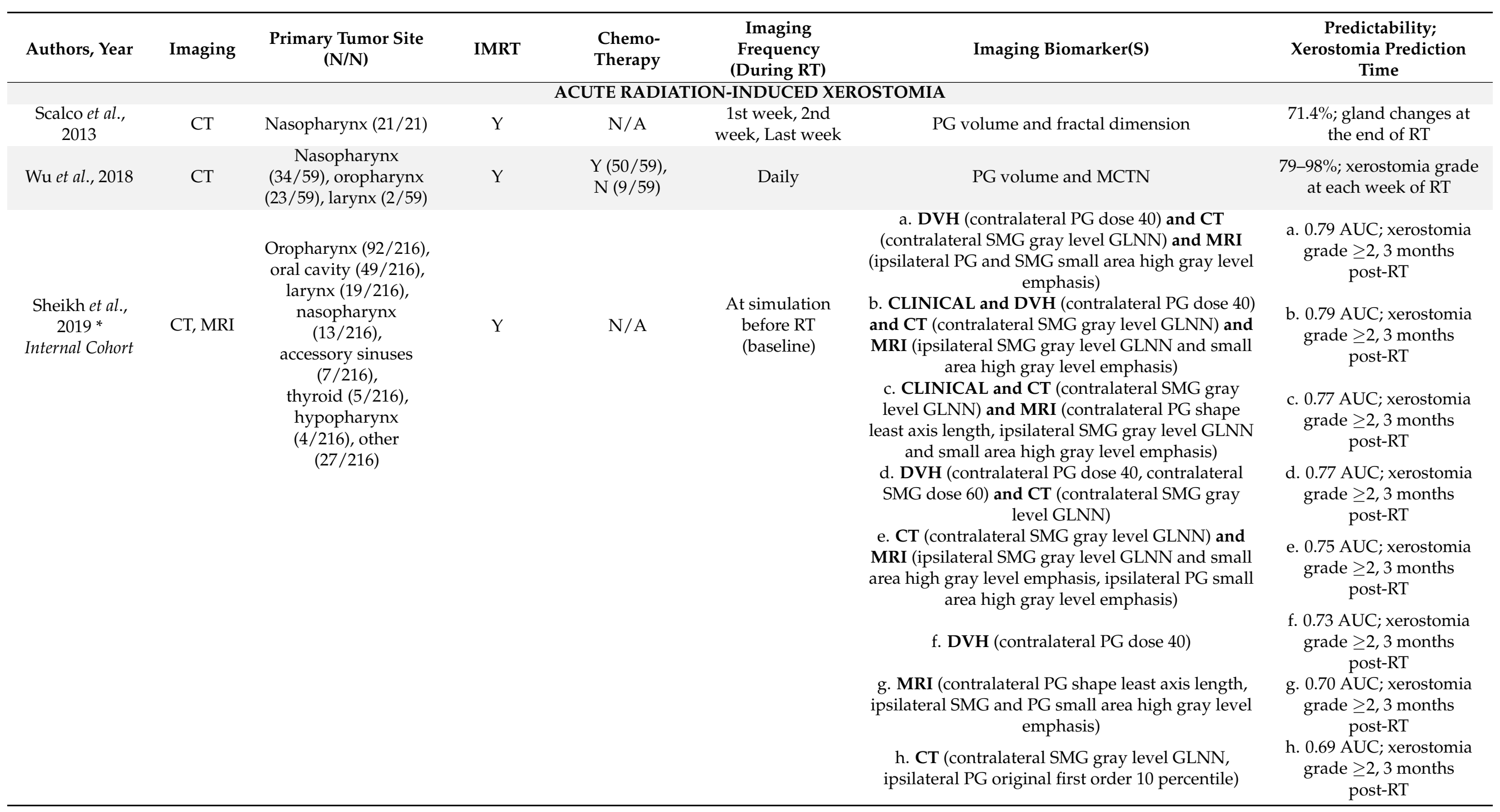


Table 1. Cont.

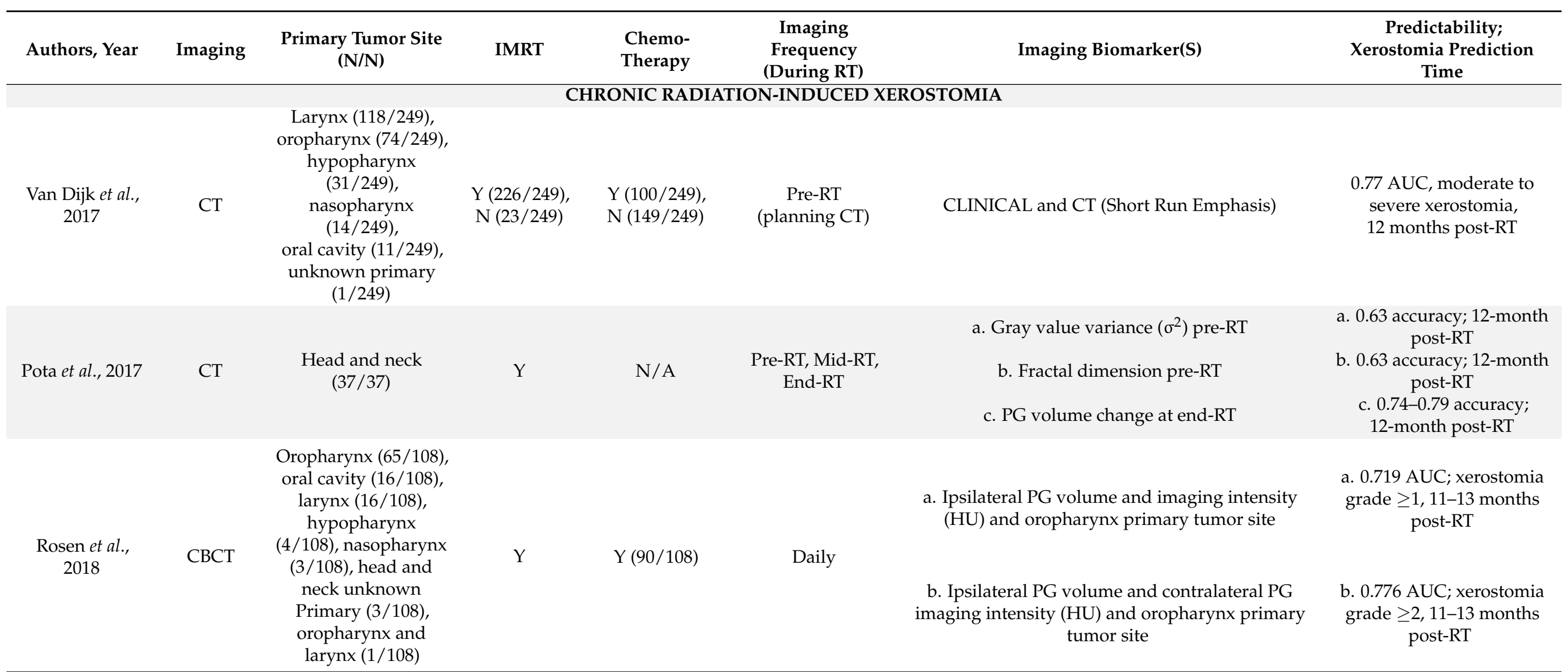

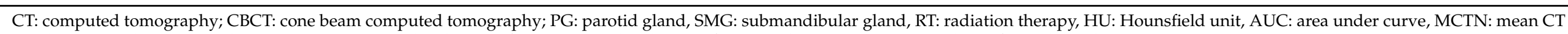
number, DVH: dose-volume histogram, GLNN: gray level nonuniformity normalized *: significant markers $p<0.05$ in parentheses for each combination. 


\subsection{Chronic Xerostomia}

The long-term xerostomia, though, can be even more important to predict, as it reflects the chronic effect of the radiation therapy on the function of the salivary glands. In this direction, imaging data were attempted to be collected throughout the radiation therapy in order for a prediction model of the xerostomia grade as observed after 11-13 months post-treatment [7]. These data derived from cone beam CT (CBCT) imaging, which is performed during the radiation therapy for the exact positioning of the patient during the consecutive radiation sessions, and, thus, no additional imaging would be needed if such a protocol can be implemented successfully. Although the study included head and neck cancer patients of various primary tumor sites (Table 1 ), the results were significant and with a relatively high predictability for the patients that had the oropharynx as the primary tumor site and combined with the parotid gland volume and intensity (Hounsfield units) changes. The xerostomia grade at 11-13 months after the radiation therapy was categorized into two groups; grade $\geq 1$ and $\geq 2$. When the aforementioned combination of imaging markers was compared to using only clinical parameters for the prediction, it had a notably increased predictability only for the chronic xerostomia grade $\geq 2$. Interestingly, significant sole factors associated with the grade $\geq 2$ xerostomia were the mean intensity loss (Hounsfield Unit) of the parotid gland of the contralateral site at weeks 4, 5, and 6 . On the contrary, no significant association with this advanced xerostomia grade range (grades 2 and 3 ) was found for the parotid glad volume change or with metrics derived from the dose-volume histograms. However, the latter metrics as derived from the ipsilateral site were significantly associated with xerostomia grade $\geq 1$.

Similarly, in another large number population of patients, the addition of pretreatment CT (planning CT) biomarkers increased the predictability of moderate to severe xerostomia as demonstrated 12 months after the radiation treatment, compared to the sole dosimetric and clinical correlation [10]. In specific, the significant CT biomarker that was found represents the heterogeneity of the salivary glands (Table 1). In this study, CT biomarkers were also found significant in the predictability of saliva alteration to "sticky saliva," which has been associated with radiation-induced side effects and xerostomia.

In another analysis of clinical, dosimetric, and CT radiomic data of a smaller population of head and neck cancer patients that attempted to identify sole, long-term (after 12 months) xerostomia predictors, the gland volume change was found as the most accurate predictor followed by the pretreatment $\mathrm{CT}$ gray scale variance and fractal dimension metrics. In this analysis, data from only three points of time during the radiation therapy were included; pretreatment, mid-treatment, and end-of-treatment [11].

The creation of a model for the long-term predictability of radiation-induced xerostomia is of undoubtful significance, as it would provide valuable information for the long-term quality of life of the patient and allow for early adaptation of the radiation therapy plan, as feasible, and early management for the patient.

\section{Combined CT and MRI}

A combination of imaging biomarkers from both CT and MRI of the parotid and submandibular glands has been attempted in order to predict the patient risk of moderate or severe radiation-induced xerostomia as observed 3 months after the completion of the radiation therapy. The imaging was taken before the beginning of the radiation therapy, during the radiation simulation. This study investigated and compared the prediction effectiveness of each imaging alone, as well as other potential predicting parameters, such as clinical data (i.e., age, sex, and tumor volume) or dose-volume histogram parameters [6]. The prediction model, tested for 216 patients, improved significantly when the parameters of CT, MRI, and dose-volume histogram were combined (Table 1). However, the addition of the three clinical parameters did not improve the model further. When the model was applied again on 50 patients for external validation the results were not decisive, but the highest model performance was achieved when CT, MRI, and clinical data were combined, followed by the combination of only the CT and MRI. 
Interestingly, as in this study model, both the parotid and submandibular glands from both sides were included, for the $\mathrm{CT}$, a contralateral submandibular gland imaging marker was one of the statistically significant markers for the model for all combinations, and not a marker of the parotid. This is a very important finding as it indicates the role of the submandibular gland in the prediction of the xerostomia side effect, and further such analyses would shed light into its role.

\section{Conclusions}

To sum up, imaging techniques such as magnetic resonance imaging and computed tomography can be powerful tools for the prediction and monitoring of radiation-induced xerostomia. The imaging techniques have been performed routinely during oncologic treatment for the identification, monitoring, and treatment of the tumor, and they can now be used as well in order to provide additional information for the prediction and prevention of the side effect of radiation therapy on the salivary glands. However, the current studies do not agree yet to a sole prediction model. In addition, the proposed imaging biomarkers of each of these studies are not safely comparable as there are multiple variables in the studied patient populations (i.e., primary tumor site, concurrent systemic treatment, etc.). To conclude, further investigation is needed in order not only to identify the most effective imaging biomarkers that are linked to radiation-induced xerostomia but also for comprehensive models to be developed.

Author Contributions: Conceptualization, investigation, and data collection, A.K. and D.K.; writingoriginal draft preparation, A.K. and D.K.; writing-review and editing, A.K. and D.K.; visualization, D.K. and A.K.; supervision, A.K.; project administration, A.K. and D.K. All authors have read and agreed to the published version of the manuscript.

Funding: This research received no external funding.

Conflicts of Interest: The authors declare no conflict of interest.

\section{References}

1. Mücke, T.; Koschinski, J.; Wolff, K.-D.; Kanatas, A.; Mitchell, D.A.; Loeffelbein, D.J.; Deppe, H.; Rau, A. Quality of life after different oncologic interventions in head and neck cancer patients. J. Craniomaxillofac Surg 2015, 43, 1895-1898. [CrossRef] [PubMed]

2. Rathod, S.; Livergant, J.; Klein, J.; Witterick, I.; Ringash, J.A. systematic review of quality of life in head and neck cancer treated with surgery with or without adjuvant treatment. Oral Oncol. 2015, 51, 888-900. [CrossRef]

3. Chaukar, D.A.; Walvekar, R.R.; Das, A.K.; Deshpande, M.S.; Pai, P.S.; Chaturvedi, P.; Kakade, A.; D'Cruz, A.K. Quality of life in head and neck cancer survivors: A cross-sectional survey. Am. J. Otolaryngol. 2009, 30, 176-180. [CrossRef] [PubMed]

4. Guibert, M.; Lepage, B.; Woisard, V.; Rives, M.; Serrano, E.; Vergez, S. Quality of life in patients treated for advanced hypopharyngeal or laryngeal cancer. Eur. Ann. Otorhinolaryngol. Head Neck Dis. 2011, 128, 218-223. [CrossRef]

5. Klein, J.; Livergant, J.; Ringash, J. Health related quality of life in head and neck cancer treated with radiation therapy with or without chemotherapy: A systematic review. Oral Oncol. 2014, 50, 254-262. [CrossRef] [PubMed]

6. Sheikh, K.; Lee, S.H.; Cheng, Z.; Lakshminarayanan, P.; Peng, L.; Han, P.; McNutt, T.R.; Quon, H.; Lee, J. Predicting acute radiation induced xerostomia in head and neck Cancer using MR and CT Radiomics of parotid and submandibular glands. Radiat. Oncol. 2019, 14, 1-11. [CrossRef]

7. Rosen, B.S.; Hawkins, P.G.; Polan, D.F.; Balter, J.M.; Brock, K.K.; Kamp, J.D.; Lockhart, C.M.; Eisbruch, A.; Mierzwa, M.L.; Ten Haken, R.K.; et al. Early Changes in Serial CBCT-Measured Parotid Gland Biomarkers Predict Chronic Xerostomia after Head and Neck Radiation Therapy. Int. J. Radiat. Oncol. Biol. Phys. 2018, 102, 1319-1329. [CrossRef]

8. Scalco, E.; Fiorino, C.; Cattaneo, G.M.; Sanguineti, G.; Rizzo, G. Texture analysis for the assessment of structural changes in parotid glands induced by radiotherapy. Radiother. Oncol. 2013, 109, 384-387. [CrossRef] [PubMed]

9. Wu, H.; Chen, X.; Yang, X.; Tao, Y.; Xia, Y.; Deng, X.; Zheng, C.; Robbins, J.; Schultz, C.; Li, X.A. Early Prediction of Acute Xerostomia During Radiation Therapy for Head and Neck Cancer Based on Texture Analysis of Daily CT. Int. J. Radiat. Oncol. Biol. Phys. 2018, 102, 1308-1318. [CrossRef] [PubMed]

10. Van Dijk, L.V.; Brouwer, C.L.; van der Schaaf, A.; Burgerhof, J.G.M.; Beukinga, R.J.; Langendijk, J.A.; Sijtsema, N.M.; Steenbakkers, R.J.H.M. CT image biomarkers to improve patient-specific prediction of radiation-induced xerostomia and sticky saliva. Radiother. Oncol. 2017, 122, 185-191. [CrossRef] [PubMed]

11. Pota, M.; Scalco, E.; Sanguineti, G.; Farneti, A.; Cattaneo, G.M.; Rizzo, G.; Esposito, M. Early prediction of radiotherapy-induced parotid shrinkage and toxicity based on CT radiomics and fuzzy classification. Artif. Intell. Med. 2017, 81, 41-53. [CrossRef] [PubMed] 